\title{
The Effective Application of Digital Piano in Piano Teaching
}

\author{
Yi Ji \\ Music and Dance College \\ Qujing Normal University \\ Qujing, China 655011
}

\begin{abstract}
With the development of piano group teaching model, the emergence of this new digital piano class teaching mode, both as a kind of piano class is different from different from the traditional class. This new teaching mode is developed on the basis of the traditional piano teaching mode, it is a derivative development of the traditional model with comprehensive, active, creative and a series of features. Group digital brought the traditional "sight singing and ear training", "impromptu" into the digital piano classroom, it is a major breakthrough for traditional piano class. Group digital Piano class effectively change the teaching environment, combine modern teaching methods and equipment together with boring theory lessons effectively, enhance the vitality of the class to mobilize the interest of students, and also greatly improve the teaching effect.
\end{abstract}

\section{Keywords—digital piano; teaching and application}

\section{Digital Piano Has a Special"Digital" Feature}

Digital Piano is not Keyboard, although it is based on 40 years of historical development for .keyboard. Digital Piano also called "electric piano" with digital technology (pulse code modulation technology) instead of the traditional analog technology. It can realize the simulation of the mechanical piano sound quality, with the full functionality of a traditional piano, but also it can achieve a variety of unique sound, mixing, tone, memory storage, metronome and other functions, as well as headphone (not caused ambient noise pollution after connected), MIDI interface and microphone interface equipment. It combined the traditional piano technology together with modern new piano technology, greatly enhanced the effect, particularly suitable for beginners.

Digital piano is not as simple as many people think just mechanical piano shell contains a keyboard core. But in fact, the Digital Piano and Key board have very big difference in essence. First of all, there is no strong or weak sound for keyboard, it is unable to control the intensity, it will sound the same no matter you play heavy or weak. But through the use of MF modulated analog technology, digital piano basically initial change this shortcoming, you can control the strength of the electronic piano sound. Secondly, the general strength level of mechanical piano is far richer than the digital piano. Mechanical piano has a sound pressure difference above $50 \mathrm{~dB}$ or more, Maximum can up to $100 \mathrm{~dB}$, and the lightest tones can reach $50 \mathrm{~dB}$, while the digital piano sound pressure difference is generally only $30 \mathrm{~dB}$ far below the mechanical piano. But digital piano technology is still in progress and improvement, the current most advanced digital piano technology has the heaviest tone of $100 \mathrm{~dB}$ and lightest tone of $30 \mathrm{~dB}$, the sound pressure difference can reach $70 \mathrm{~dB}$ or more, which is much higher than the traditional mechanical piano. And digital piano can implement a uniform transition volume within the whole tone range with different sound performance, fully achieved free expression of emotional ups and downs.

Digital Piano use digital technology instead of analog technology, so timbre distortion will not exist, it effectively realizes the simulation of the traditional mechanical piano. Digital piano and traditional mechanical piano played in the same sound effects. First the digital piano can simultaneously achieve 88 key independent utterances on the diatonic harp; second the digital piano is able to control the sound, play heavy the sound will be heavy, play weak the sound will be weak. When play short sound has attenuation shaped extension, when play long sound it has attenuation shaped extension tone; third the static resistance change of digital piano full keyboard also fully meet the required standards. In addition, the digital piano sound is excellent with stable performance to achieve a breakthrough on some of the features and characteristics. First is it achieves sounds height adjustment function with full digital piano tone with the difference between high and low tones plus or minus 50 cents and it will greatly improve the synergistic effect when played with orchestra; The digital piano is able to played with all seasons and at all places without affect others people's rest. In addition, the digital piano also adopt the internationally accepted standard MIDI interface, you can connect to your computer and use ; The third is the digital piano has a good price, traditional mechanical piano easily cost several hundreds thousand dollars, while the digital piano only cost half or even eighth of the price of a traditional piano, truly is affordable. I believe that in the near future, a large number of modern digital pianos will be launched in the market to meet the demand for more piano fans and achieve universal access in China for piano and music education.

\section{The Digital PiAno CAN EFFeCtively Optimize THE ALlOCATION OF TEACHING RESOURCES}

All the time piano teaching methods are basically one-toone no matter school education, social education or family 
education, it normally shows teacher guidance, students practice mode. In this teaching method, students can get fully detailed learning to improve their personal skills steadily. Piano teachers can make the appropriate guidance depending on the student's personality and characteristics. Therefore, this one-to-one teaching method has been considered the only effective way to specialized teaching piano, music teaching faculty in higher education in this regard, with no exception. The traditional piano teaching mode is arising and developing based on the teaching objectives of professions and skills, but also is the inevitable result under the lack of teaching resources in socio-economic conditions.

In recent years, along with the continuous development of social economy and culture, whether it is family or community shows more and more attention for piano and other artistic training, more and more people start to learn piano, the number of piano students have also increased year by year, but the number of piano teachers did not match the increasing pace with the students, so teaching workload is heavy and resulting in a gap between the quality of teaching and the number of students which is difficult to solve. The traditional teacherstudent one-to-one mode can not meet the real needs because the number of piano teachers is difficult to increase in a short time and also training a great number of piano teachers is high cost. Aiming at the current difficulties, according to the characteristics of music professional learning and piano teaching, group digital piano teaching is an effective solution. We can solve the urgent problem with lack of piano teachers on one hand and on the other hand this solution can optimize the allocation of teaching resources in schools and the whole society, this solution will produce significant benefits for sure.

Piano Lessons for Music Department of Higher Education is a compulsory foundation course, the goal of this course is to take the piano as a complementary tool in the future teaching work and other professional courses, it is not to cultivate highlevel pianist. I believe that piano lessons for non-piano major students can use digital piano for teaching. Training objectives for students in non-piano major are capable of the preschool and school music teaching in the future, we need to focus on the accompaniment capabilities which do not require a high level of culture as a pianist. The pianist need one-to-one teaching mode with a lot of manpower and resources. Take digital piano group teaching mode can effectively achieve teaching objectives, but also save the investment of resources.

In pre-school and school piano interest teaching, in addition to a small part of students who has talent and determined to be a professional pianist, most of the students learn the piano as a talent and interest, therefore do not need all learners use the one-to-one teaching mode. The majority of learners can take the digital piano group teaching mode, on the one hand to solve the problem of shortage of teachers, on the other hand can also reduce tuition for students' family burden, allowing more students begin to learn piano, it is also an effective balance between teaching objectives and resources investment.

In short, the digital piano group teaching mode broke the traditional piano teaching one-to- one mode, on the one hand it helps save teaching resources and solve the shortage of teachers, on the other hand it also improves the teaching efficiency in order to meet more piano teaching demands.

\section{The Confusions In Digital Piano TEACHING}

Although digital piano has many advantages, it can effectively optimize the allocation of resources and there are already digital piano classrooms in the music faculties of higher education schools. But we also we need to see indeed in reality, there are some confusions for this teaching mode and this confusions are the difficulties to change people's attitude to digital piano teaching.

\section{A. Whether the Digital Piano Teaching Can Match the Traditional Piano}

Digital piano do has some differences about sound quality and tactility compare with traditional piano, some piano teachers have less contact with digital piano, which lead them believe that the practice result of the digital piano can not be as good as traditional piano.

For this problem we should take a rational view. First, on the touch-key tactility and sound quality, digital piano do exist some differences with traditional piano, the differences include the piano type and structure, but also include the psychological different perception caused by their unfamiliar and no adapt with the digital piano. There is a process of adaptation for every new thing, I believe that after a period of use and practice, you can quickly get the advantages of digital piano. Each traditional piano is not the same, replace the piano also need some time to adapt. Even play on the same piano, we must continually adjust the touching way and tactility. Famous American expert Professor Hannah, an expert who has made remarkable achievements in both the traditional piano teaching and digital piano group teaching, considered there is no much difference between traditional and digital piano on key touch, she believe the key is how to use, not the piano category.

Traditional piano is heavier than digital piano and require frequent tuning. Although digital Piano at present can not completely simulate the traditional piano sound quality and tactility effect, it is a musical instrument still in its development, I believe that with the continuous development of modern science and technology, the sound quality and tactility of digital piano will have a breakthrough improvement not only can fully simulate the traditional piano sound quality and tactility, and may even achieve more and more powerful hardware and software functions and more colorful sound changes.

\section{B. Whether the Group Teaching Mode Can Guarantee the Teaching Effect}

Some piano teachers are doubtful about the group teaching mode, they believe group teaching can not achieve the teaching effect they want. Group teaching mode is not as good as the one-to-one teaching mode on instruction in the fine degree. But this issue should be considered rationally. First, teaching effect is good or bad depends on many factors and should not be simply attributed to a single cause of teaching mode, the ability of teachers, the quality of students, how much time for practicing, practicing quality level, the state of 
cooperation between teachers and students, all there factors are likely to influence the teaching effect, teaching mode is one aspect, but not all. Excellent piano teachers may obtain good teaching effect through group teaching, bad piano teachers may also get bad effects even through one-to-one teaching mode. It can not be simply rudely considered that one-to-one mode is better than group teaching mode.

Strictly speaking, one-to-one mode and group teaching mode both have advantages and disadvantages, the two can complement each other. They both have their exist reasons and one can not replace another. The proper relationship between the two should complement each other, With the increasing number of piano learners, if all use one-to-one teaching mode, apparently teachers are not enough, their workload will has a significant increase so teaching effectiveness can not be guaranteed.

All the time the choice for teaching mode has closely related with training objectives and requirements. China's famous music educator Mr. Zhao Feng had pointed out, the Normal Schools are always compare to music institutions which paid more attention in the specialized training. Some students can play difficult solos, but can not play impromptu, so they can only do solo performance can only do solo performances not stage accompaniment, so they can not serve as a music teacher in primary and secondary schools. As previously described, Piano Lessons for Music Department of Higher Education is a compulsory foundation course, the goal of this course is to take the piano as a complementary tool in the future teaching work and other professional courses. Therefore, digital piano group teaching in the Piano Lesson in Higher Normal Education is regarded as both efficient and effective way of teaching and it could promote students' comprehensive quality and practical ability to work. The advantages of modern digital piano group teaching can effectively solve the problem of piano teacher resource shortage, greatly save the teaching cost.

\section{The CuRrent Situation OF the Digital Piano GROUP TEACHING MODE}

At present, China's digital piano group teaching is still in the exploratory stage and keep progressing. The hardware and software of Digital Piano functions still need further exploitation, and it has not been able to establish a complete system of teaching methods and materials system. Most group teaching teachers still use one-to-one teaching mode teaching material and methods so it failed to achieve a significant improvement in teaching effect, also failed to fully demonstrate the advantages of group teaching mode. This is an important reason why people are doubt with the digital piano group teaching mode. The development of things has a certain process, the development of digital piano group teaching has also following the agreed law. With the promotion and popularization of this new piano teaching method, teaching materials and teaching system will be more perfect, the utility of this new method by piano teachers will be more skilled, and I believe we will have more objective and rational evaluation for the digital piano group teaching then. And for now, digital piano group teaching is like a small tree which needs our more careful nurturing and sum up the experience to achieve a breakthrough.

\section{THE EXPECTED EFFECT OF THE Digital PiANO GROUP TEACHING}

\section{A. New Digital Piano Group Teaching Mode Is More in Line with the Training Objective for Normal University}

Digital piano group teaching mode is stronger in comprehensiveness, creativeness and practicalness, it emphasis more on practical ability training in order to help to improve the comprehensive quality of students, enhance the working ability rather than just focusing on playing skill. Digital piano group teaching mode enables to be thoroughly acquainted with music knowledge and skills. Students with no piano base can also learn to do piano accompaniment through short time group teaching lessons. When engaged in pre-school, primary and secondary music education, they are able to do solo singing and also improvised accompaniment to enhance the teaching ability and skills. It will greatly change the previous situation which teachers can only do solo but not able to do piano accompaniment.

\section{B. New Digital Piano Teaching Mode Can Break the Shackles of Teaching Ideas}

It can be seen that in the future the professional knowledge and structure of digital piano teaching mode will be changed and the piano teachings level be will greatly enhanced, the piano teaching theoretical system will also be more abundant. In the past one-to-one teaching mode did not systematized Piano Teaching System. New digital piano teaching mode has brought a new direction to the piano teaching research Piano teacher must face the traditional values and modern technology developments, they should change their attitudes and sum up the experience and theory in order to ensure the high quality of piano teaching, and also can adapt to the development of technology and balance the teaching effectiveness with the resource utility.

\section{New Digital Piano Teaching Mode Can Effectively Improve Teaching Efficiency}

As previously mentioned, the piano learners become more and more, digital piano teaching mode will greatly liberating the teaching productivity and greatly enhance the efficiency and effectiveness of teaching. Digital piano group teaching is fully able to achieve centralized explain common knowledge, group practice for the required skills, it can avoid duplication of teaching in order to achieve significantly workload reduction and saving teaching time. At the same time, it will greatly enhance the operating efficiency to meet the actual needs of college enrollment expansion.

\section{CONCLUSION}

Digital piano group teaching is a new teaching mode emerged by economic, social and technology continues development and it is a major breakthrough for the traditional one-to-one teaching mode. On one hand it learned the educational thoughts of music education and teaching 
principles from traditional piano education, on the other hand it followed the trend and development of modern education reform, fully use the results of modern scientific and technological developments, combine the piano education together with modern technology effectively. I believe in the future piano education will be more scientific and healthy development toward a better direction, it will make more useful results.

\section{REFERENCES}

[1] "The New Digital Piano Group Teaching Mode" Zhou Yong, "Journal of Hunan City University", first issue in 2006.

[2] "Change of Piano Teaching Concept" Li Bingsen, "Data of Culture and Education", 20th issue in 2006.

[3] "Shallow Knowledge with Digital Piano Group Lesson", Wu Ting, "Explorations in Music",1st issue in 2006.

[4] "Foreground Application Analysis of Digital Piano Teaching", Wan Liping, "Dajiang Weekly (Forum)"9th issue in 2009.

[5] "Advantage of Digital Piano Groupteaching Compared with Traditional Piano Teaching" An Jing, "Beauty and Times", 7th issue in 2004 